Education of Medical experts. However, the level of knowledge of Truvada PreP was proportional to their Education.

Conclusion The survey showed that more information on Truvada PreP usage should be disseminated since its usage still remains a myth to most Kenyans (there is still a strong believe that AIDS as no cure).Therefore, for a successful Truvada usage depends on Opinion leaders, health Professionals and researchers to correct the current misconceptions existing about Truvada PreP in their communities

\section{P5.09 AWARENESS OF HIVIAIDS AND STI'S AMONG VISUALLY IMPAIRED FEMALE SEX WORKERS (VIFSWS) IN ELDORET TOWNSHIP, KENYA}

Charles Salil. Information Centre on HIV Prevention, Department of Research and Sexology, Kenya

\subsection{6/sextrans-2017-053264.625}

Introduction In the recent past, it has been a notion that VIFSWS have a greater risk of acquiring HIV due to their Physical challenge they face.Howver,according to a report from UNAIDS which says that women have a greater risk of becoming infected than men if they are unprotected. The aim of the study is to analyse the level of awareness about HIV/ AIDS/STIs among VIFSWs from their Brothels in Eldoret Township, Kenya.

Methods A descriptive study was conducted where Qualitative methods were used. In depth Interviews was conducted on 60 VIFSWs and out of those 60 ,a strata of 6 was formed. The data was then put on codes and later interpreted.

Results According to the results, it was found out that 99\% of the respondents in the study had knowledge on HIV/AIDS and STIs.However, the majority of the respondents faced ill treatment by their male clients due to their physical challenge they face. Also they faced stigma and discrimination from members of Public, on availability of user friendly health care services was also another challenge. They also lacked Testing and Counselling services from government Institutions.

Conclusion According to data obtained from the field, it suggests that the level of awareness of HIV/AIDS/STIs is high among the VIFSWs. However, there is an urgent need to conduct regular Counselling and Testing since they have less knowledge on importance of Counselling and Testing and early use of PeP in case of an exposure or early use of Anti Retroviral Therapy (ART).

\section{P5.10 INHIBITORY EFFECT OF CHLORHEXIDINE ANTISEPTIC MOUTHWASH AGAINST NEISSERIA GONORRHOEAE? AN IN-VITRO STUDY}

Chris Kenyon, Vicky Cuylaerts, Tania Crucitti. Institute of Tropical Medicine, Antwerp, Belgium

\subsection{6/sextrans-2017-053264.626}

Introduction Studies have found that oral sex plays a major role in the transmission of Neisseria gonorrhoeae (NG) in men who have sex with men (MSM) populations. We aimed to establish in vitro the concentration and exposure time of Chlorhexidine mouthwash (Corsodyl â) that can inhibit the growth of NG to less than 102 Colony Forming Units (CFU) per $\mathrm{ml}$.
Method Four NG strains isolated from pharyngeal specimens were selected for this exercise. Three were isolated from women, and all four were susceptible to ceftriaxone, cefixime, and spectinomycin, three were less susceptible (intermediate) to azithromycin and two were resistant to ciprofloxacin. None of the strains produced penicillinase. The antibiotic susceptibility was obtained using the agar dilution method and European Committee on Antimicrobial Susceptibility Testing breakpoints were used. Of each of the isolates a suspension of approximate $108 \mathrm{CFU} / \mathrm{ml}$ (0.5 Mc Farland) was prepared in Phosphate-Buffered Saline (PBS)(positive control) and in serial dilutions of Chlorhexidine in PBS- $0.2 \%, 0.1 \%$ and $0.05 \%$. Following 30 and $60 \mathrm{~s}$ of exposure at ambient temperature a volume of $10 \mu \mathrm{l}$ of each of the suspensions was plated onto BBLTMColumbia blood agar (5\% horse blood) and incubated for 48 hours $\left(5 \%-7 \% \quad \mathrm{CO} 2,35 \pm 2^{\circ} \mathrm{C}\right)$. The colony growth was recorded and the number of CFU was counted, if appropriate. All experiments were conducted in triplicate.

Results Abundant growth was obtained with all PBS control suspensions. Zero CFU/ml were retrieved for all experiments using $0.2 \%$ chlorhexidine and $60 \mathrm{~s}$ of contact time. In only one of the 12 experiments using $0.2 \%$ chlorhexidine and $30 \mathrm{~s}$ of contact time a NG growth of $100 \mathrm{CFU} / \mathrm{ml}$ was obtained. Lower concentrations of chlorhexidine inhibited the growth of NG but to a lesser degree than $0.2 \%$. The longer contact time inhibited the growth more frequently compared to the 30 s of contact time.

Conclusion The efficacy of the inexpensive and widely available $0.2 \%$ chlorhexidine mouthwash in preventing or treating pharyngeal NG merits consideration in clinical trials.

\section{P5.11 ASSOCIATION OF HUMAN MANNOSE RECEPTOR(HMR) IN SEXUAL TRANSMISSION AND PATHOGENESIS HIV SUBTYPE C VIRUS IN SERODISCORDANTS COUPLES}

${ }^{1}$ Shivaji Kashinath Jadhav, ${ }^{2}$ Bandivdekar AH. 'National Institute for Research in Reproductive Health [NIRRH], ICMR and Sandor Life Sciences; ${ }^{2}$ National Institute for Research in Reproductive Health [NIRRH], ICMR, India

\subsection{6/sextrans-2017-053264.627}

Introduction HIV binds specifically to human Mannose Receptor (hMR) on vaginal epithelial cells which are devoid of conventional CD4 receptor. HIV binding to hMR on vaginal epithelial cells induces the production of Matrix Metalloproteinase 9 (MMP9) leading to degradation of extracellular matrix which may increase the risk of sexual transmission of HIV. Methods PCR amplification of DNA from PBMCs of the serodiscordant females for CCR5 gene flanking for CCR5-delta 32 region. Translated amino acid sequence of $\mathrm{C} 2-\mathrm{V} 3$ region of env gene of HIV PBMCs and sperm of the infected male partners of the Serodiscordant couples was determined. The localization of hMR on vaginal epithelial cells of the seronegative females from general population and seronegative females from Serodiscordant couples was studied using FITC labelled antibodies to hMR (FITC AbhMR).

Results Translated amino acid sequence of $\mathrm{C} 2-\mathrm{V} 3$ region of env gene of HIV1C in PBMCs $(n=9)$ and sperm $(n=5)$ of the male partners showed the presence of distinct variants and the variation in PBMCs and sperm of serodiscordant males was almost similar to that of infected males from concordant couples. The Presence of hMR on $0 \%-11 \%$ of the vaginal epithelial cells of seronegative females $(n=39)$ from serodiscordant couples and 90\%-95\% that of control group of females. 
Conclusion The study suggests the association of hMR in sexual transmission of HIV. Presence of hMR in lower number of vaginal epithelial cells of Serodiscordant females prevented binding and HIV entry into these cells.

\section{P5.12 KNOWLEDGE AND AWARENESS AMONG CERVICAL CANCER PREVENTING VACCINE AMONG WOMEN IN SUBURBAN AREAS IN SRI LANKA}

Eashaan Maneendra Dassanayaka, EASI Edrisinghe, KAMJ Perera, WGWGC Bandara, SN Silva. International Institute of Health Sciences, Sri Lanka

\subsection{6/sextrans-2017-053264.628}

Introduction Cervical cancer is the 2nd most common cancer in the world whilst the incidence of cervical cancer continue to rise in Sri Lanka. It's important to assess the knowledge and the awareness on the cancer and the HPV vaccine. The Objective of this research was to assess the knowledge and awareness about the cervical cancers and HPV vaccine.

Methods A descriptive cross sectional study was done on 326 Sri Lankan urban and sub-urban females using convenient sampling who were in the age between 14-39 years using a self-administered questioner.

Results $(62.9 \%)$ of the participants, were from the age group 21-29. (50.8\%) had an advance level education, 30.4\% had an undergraduate level education. (55\%) haven't heard about a vaccine which could prevent cervical cancer, 51.2\% knows that cervical cancers are common in Sri Lanka. 193 (59.2\%) did not know that HPV is the most common cause for cervical cancers. When considering occupational level $(p<0.001)$ and and education level $(\mathrm{p}=0.001)$ it played a key role in determining the awareness of HPV vaccine on women. Respondents from the government sector with a good education had more awareness on the HPV vaccine. 28\% obtained information on HPV vaccine through health care professionals or the Internet. Only 4\% have been vaccinated and reason for not getting a vaccine is due to lack of knowledge. Only $11.3 \%$ knew about the correct does for the vaccine.

Conclusion It's evident that the knowledge and awareness is very low in terms of the vaccine.

\section{P5.13 WILLINGNESS TO USE PRE-EXPOSURE PROPHYLAXIS AMONG FEMALE SEX WORKERS IN THE MEXICO- UNITED STATES BORDER REGION}

${ }^{1}$ Heather Pines, ${ }^{1}$ Strathdee AS, ${ }^{2}$ Hendrix CW, Bristow CC, ${ }^{3}$ A Harvey-Vera, ${ }^{1}$ MagisRodríguez C, ${ }^{4}$ Martinez G, ${ }^{1}$ Semple SJ, ${ }^{1}$ Patterson TI. ' University of California, San Diego, USA; ${ }^{2}$ Johns Hopkins University, USA; ${ }^{3}$ Centro Nacional Para La Prevención y Control Del VIHISIDA (CENSIDA), Ministry of Health, Mexico; ${ }^{4}$ Federacion Mexicana de Asociaciones Privadas, Mexico

\subsection{6/sextrans-2017-053264.629}

Introduction We assessed willingness to use pre-exposure prophylaxis (PrEP) to prevent HIV among female sex workers (FSWs) in Tijuana and Ciudad Juarez, two northern Mexico cities bordering the United States (US).

Methods To date (7/2016-12/2016), 279 HIV-negative FSWs in a behavioural HIV prevention intervention trial have tested for sexually transmitted infections (STIs) and completed surveys to assess (1) preferences between and willingness to use 12 hypothetical PrEP products with varying attributes with respect to formulation (pill, gel, douche, or ring), frequency of use, cost, effectiveness, side effects, and access point and (2) perceived motivators and barriers to PrEP use. Fisher's exact tests were used to examine differences in willingness to use preferred PrEP products by STI diagnosis and sociodemographic, behavioural, and sex work characteristics.

Results 94\% (263/279) of FSWs were willing to use their preferred PrEP product. Willingness to use preferred PrEP products was lower among FSWs diagnosed with an STI $(83 \%$ vs. $97 \% ; \mathrm{p}=0.001)$, living in Tijuana (88\% vs. 99\%p<0.0001), primarily practicing sex work on the street $(89 \%$ vs. $97 \%$; $\mathrm{p}=0.01)$, reporting methamphetamine use (past month) $(86 \%$ vs. $97 \% ; \mathrm{p}=0.001)$, and reporting hazardous alcohol consumption (past year) $(91 \%$ vs. $97 \% ; \mathrm{p}=0.04)$. Of the 16 FSWs unwilling to use PrEP, "PrEP does not provide full protection against HIV" was the most common perceived barrier to PrEP use $(88 \%)$ followed by "I would have trouble using PrEP consistently because of my alcohol use" (63\%) and "I am worried I will lose clients if I use PrEP because they will assume I am HIV-positive" (63\%). All FSWs unwilling to use PrEP reported that additional protection against other STIs would motivate them to use PrEP.

Conclusion PrEP interest was high among FSWs along the Mexico-US border. Our findings suggest that the development of multi-purpose PrEP products and site-specific interventions that provide PrEP education and address substance use and clients' perceptions as barriers to PrEP use may support FSWs' future PrEP uptake.

\section{P5.14 AUTOIMMUNE DISEASES IN HIV-INFECTED PATIENTS}

1,2 Hejer Harrabi, 1,2Sameh Aissa, 1,2Rim Abdelmalek, ${ }^{2}$ Badreddine Kilani, 1,2 Lamia Ammari, ${ }^{2}$ Fakher Kanoun, ${ }^{1,2}$ Ahmed Ghoubontini, ${ }^{1,2}$ Hanene Tiouiri Benaissa. 'La Rabta University Hospital, Tunisia; ${ }^{2}$ Tunis- El Manar University, Tunisia

\subsection{6/sextrans-2017-053264.630}

Introduction Autoimmune and systemic diseases (ADs) were described in HIV infected patients and a classification by immune status was proposed.

Methods HIV-infected patients that presented an $\mathrm{AD}$ in the infectious diseases department of La Rabta University hospital in Tunis (Tunisia) were retrospectively included.

Results Four patients were included. The ADs were spondyloarthropathy, Behçet disease and psoriasis. Two patients presented Behçet disease. In two patients, the AD preceded HIV infection and in the two others, HIV infection was diagnosed at the same time as the $\mathrm{AD}$. In all cases, $\mathrm{ADs}$ occurred in patients with a CD4 T lymphocyte count of more than 200/ $\mathrm{mm}^{3}$. No co-infection with hepatitis $\mathrm{B}$ or $\mathrm{C}$ viruses was diagnosed.Three patients received anti-inflammatory drugs and one patient received immunosuppressant treatment with good tolerance.

Conclusion $\mathrm{AD}$ and autoantibodies are present in HIV infection. AD may develop during acute viral infection (Stage I), with normal to low CD4 counts (Stage II). However, past a threshold where the CD4 count is profoundly low, AD cannot develop (Stage III). Following HAART, immune restoration (normal CD4 count) with possible altered immune regulation may lead to the emergence of AD (Stage IV). More studies are necessary to identify the subgroups of HIV-infected patients that may be prone to develop AD. Co-infection with hepatitis $\mathrm{B}$ or $\mathrm{C}$ viruses should be screened. 